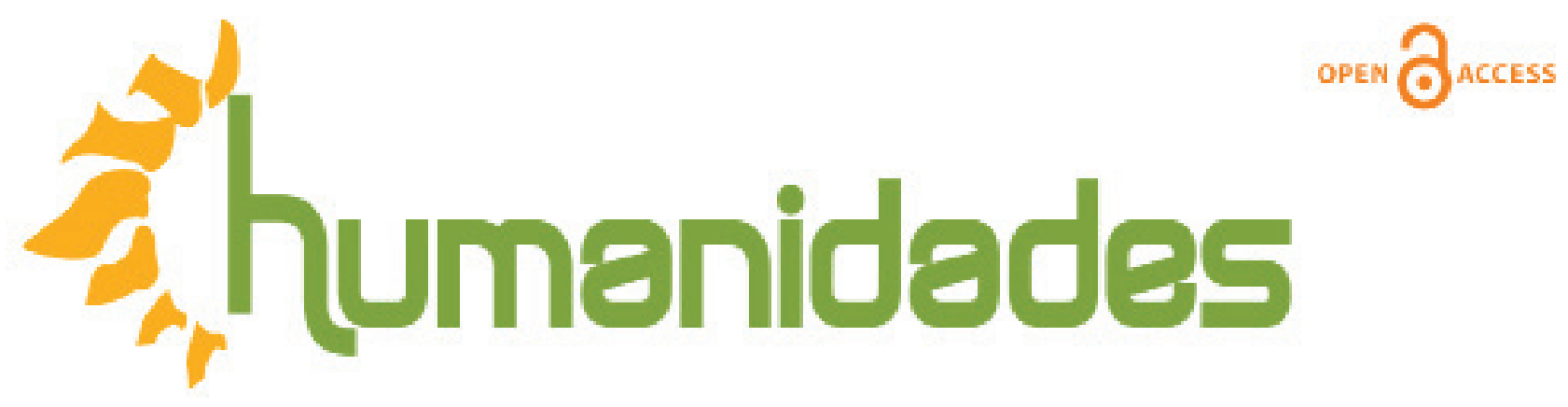

Revista de la Escuela de Estudios Generales, Universidad de Costa Rica

Enero-julio, 2019 •Volumen 9, número 1 • EISSN 2215-3934 • pp. 1-26

Recibido: 15-Octubre-2018 Aceptado: 14-Noviembre-2018

\title{
Polarización y demonización en la campaña presidencial de Colombia de 2018: análisis del comportamiento comunicacional en el Twitter de Gustavo Petro e Iván Duque
}

DOI: https://doi.org/10.15517/h.v9i1.35343

\section{Óscar Andrés Prada Espinel}

Máster en Ciencias Sociales, Universidad Internacional de Andalucía, España.

\section{Luis Miguel Romero Rodríguez}

Doctor en artes, profesor de la Universidad Internacional de la Rioja, España.

Correo electrónico: oscar.prada824@alu.uhu.es / luismiguel.romero@unir.net

Todos los derechos reservados. Universidad de Costa Rica. Esta revista se encuentra licenciada con Creative Commons. Reconocimiento-NoComercial-SinObraDerivada 3.0 Costa Rica. Correo electrónico: humanidades@ucr.ac.cr/Sitio web: http: //revistas.ucr.ac.cr/index.php/ humanidades 


\section{Polarización y demonización en la campaña presidencial de Colombia de 2018: análisis del comportamiento comunicacional en el Twitter de Gustavo Petro e Iván Duque}

\section{Resumen}

La plataforma digital Twitter se ha vuelto para los candidatos presidenciales una herramienta fundamental para generar un acercamiento con la ciudadanía y expresar las bases fundamentales de su plan de acción en caso de ser elegidos. El presente trabajo tiene como objetivo analizar la comunicación política a través de Twitter durante la campaña electoral hacia la presidencia de Colombia en el año 2018. A partir de una investigación de diseño cuali-cuantitativo, de corte descriptivo y alcance correlacional, se analizaron las cuentas de Gustavo Petro (@PetroGustavo) e Iván Duque (@IvanDuque) durante el mes previo a la primera vuelta y los veintiún días previos a la segunda vuelta del proceso electoral. Específicamente, se examinó el número de seguidores que ostentaban estos perfiles y, en cuanto al contenido de estos, se indagó cuáles fueron los temas en los que hicieron hincapié los candidatos.

\section{Polarization and Demonization in the 2018 Presidential Campaign of Colombia: Analysis of Twitter's Communication Behavior by Gustavo Petro and Iván Duque}

\begin{abstract}
This research aims to analyze political communication through Twitter during the presidential election campaign in Colombia in 2018, since this digital platform has become an essential tool to generate a rapprochement with the citizenship and express the fundamental basis of its action plan if elected. Based on a qualitative-quantitative, descriptive and correlational design research,

the accounts of Gustavo Petro (@petrogustavo) and Iván Duque (@IvanDuque)were analyzed during the month prior to the first round and the 21 st days before the second round in the electoral process. To do this, the number of followers who held these profiles was examined and what were the topics in the contents that the candidates emphasized.
\end{abstract}

Palabras clave: ciberpolítica, Twitter, Colombia, elecciones, comunicación política, ciberdemocracia

Keywords: ciberpolítica, Twitter, Colombia, elections, political comunication, ciberdemocracy 


\section{Introducción}

En Colombia se atribuye el inicio del uso de las redes sociales en campañas electorales cuando en el año 2010 el Partido Verde liderado por Antanas Mockus, candidato presidencial en ese momento, consolidó el movimiento «Ola Verde» que dinamizó la injerencia de las redes sociales como eje de su campaña, orientándola a las acciones comunicativas de determinados colectivos juveniles, visibilizadas como un maremágnum emocional e iconosférico impulsivo en la exigencia de cambios (García-Dussaán, 2011). Después de esas elecciones, todos los candidatos le han dado a las redes sociales -en especial a Twitter- un papel protagónico en la difusión de contenidos durante sus campañas.

En el transcurso de la campaña presidencial de Colombia del año 2018, las cuentas de Twitter de los candidatos sirvieron de ventana digital para que los posibles electores conocieran varias de sus posturas ideológicas y planes de gobierno. Por lo que se puede establecer que Twitter es utilizado por los líderes políticos colombianos para "construir relaciones más cercanas con los usuarios, en las que pueden exponer sus opiniones dirigiéndose de una manera más directa a las audiencias" (Barreto, López y Sánchez, 2013, p.293) e, igualmente, permitir que las personas tengan una participación frente ellos como los líderes, dado que por medio de sus publicaciones se expresan y tienen la posibilidad de ser leídos por aquellos que antes eran inaccesibles (Barreto et al., 2013).

Para esta investigación fue imprescindible analizar el comportamiento comunicacional de las cuentas de Twitter de los candidatos Iván Duque (@) IvanDuque), del partido Centro Democrático, y Gustavo Petro (@petrogustavo), de Colombia Humana, durante los períodos del 27 de abril al 27 de mayo y del 28 de mayo al 17 de junio. Se debe tener en cuenta que la Constitución Política de Colombia (1991) consagra que la elección del presidente y del vicepresidente se hará por mayoría absoluta; lo cual significa que el día de los comicios el candidato 
que obtenga la mitad más uno del total de los votos sufragados será elegido presidente de la república, pero si ninguno logra ese resultado, se celebrará una nueva votación tres semanas más tarde, conocida como «segunda vuelta electoral», en la que solo participarán los dos candidatos que hubiesen obtenido las votaciones más altas.

\subsection{Ciberdemocracia y redes sociales}

Desde sus orígenes en la antigua Grecia, el concepto de democracia ha evolucionado alcanzando un mayor nivel de comprensión con las revoluciones burguesas de Francia, Alemania y Estados Unidos, alimentando su complejidad a partir del contraste de los diferentes pensamientos hasta instaurarse como el mejor sistema político y de organización social, en el cual el poder pertenece y se encuentra legitimado por el demos (pueblo) para determinar su vida política, situando a las personas en un plano de igualdad intrínseca (Dahl, 1992), presentando algunas modificaciones y variantes, como la democracia directa, participativa, parcial y representativa, dependiendo de cada país y el tipo de organización del Estado; pero conservando las garantías en forma de derechos y oportunidades que favorecen la protección de los intereses y de la libertad de las personas (Dahl, 1992).

La aparición de la web 2.0 replanteó este concepto y lo adaptó a las características que ofrece una sociedad interconectada, puesto que no es posible reinventar los instrumentos de la comunicación y del pensamiento colectivo sin reinventar la democracia (Levy, 2004). Es entonces cuando se habla de «ciberdemocracia», "un proceso poliárquico y multidimensional que se construye y desarrolla en el espacio ciber-digital, como reflejo y expresión prospectiva de la democracia real" (Galleguillo, 2016, p.82) que, a través de las herramientas y canales tecnológicodigitales, especialmente las redes sociales, genera ciberactivismo por parte de los tecnociudadanos.

La ciberdemocracia "integra la praxis política con lo formal a través de la disponibilidad mediática, con lo sustantivo mediante la convergencia sinérgica y con los resultados por la predisposición política” (Galleguillo, 2016 p.305), confiriendo 
a las redes sociales el rol de un espacio discursivo-deliberativo en el que "también se aumentan y profundizan las parcelas o burbujas ideológicas, trasladando el discurso público y los enfrentamientos polarizantes a estas esferas" (RomeroRodríguez, Aguaded y Gadea, 2015, p.105). Por ello los líderes políticos y, en consecuencia, sus seguidores, las han convertido en un campo de batalla donde las disputas se basan en la desacreditación de todo aquel que no esté a favor de sus planteamientos, y de este modo lograr la fragmentación de la sociedad. Dicha fragmentación es el resultado de una estrategia de manipulación que consiste en la construcción de grupos sociales aislados quienes se declaran la guerra entre sí y adquieren una condición dual de víctimas y victimarios impidiendo la construcción de mayorías hegemónicas (Binder, 1992); un juego de divide et impera que a través de las redes sociales y el discurso empleado en ellas apela a la agrupación de usuarios con características, intereses o aspiraciones comunes o relacionadas.

Este binomio, víctimas-victimarios o protagonista-antagonista, "no solo busca una identificación social en relación con el poder con el objetivo de obtener obediencia, sino también crear un culpable -histórico o actual- de los desaciertos gubernamentales en ejercicio" (Romero-Rodríguez et al., 2015, p.104). Un método efectivo utilizado para este fin es la divulgación de noticias falsas (fake news), puesto que su creación es más rápida y barata que las reales sucede que, o bien los usuarios no las dilucidan con exactitud o bien ciertos consumidores solo disfrutan de la información partidista que desfavorece al contrario (Allcott y Gentzkow, 2017), lo cual distorsiona la realidad y genera un entorno de «posverdad» entorpeciendo la democracia y por extensión la ciberdemocracia.

Lo descrito ut supra crea, en "una sociedad donde cada vez es más profunda la sensación de empobrecimiento de la realidad" (Bauman, 2002, p.95), un cúmulo de falsas percepciones del entorno debido a la intoxicación, un proceso que surge a raíz del bombardeo constante de información que genera una dilación de la realidad $\mathrm{y}$, posteriormente, una sobresaturación cognitiva y distracción inminente por la ausencia de infodietas y educación en competencias mediáticas de las audiencias (Romero- Rodríguez et al., 2015, p.155). 


\subsection{Discurso Político y redes sociales}

Para Fairclough (1989), el discurso es un proceso en el que, además del texto (contenido), se incluye el proceso de producción del que el texto es producto y el proceso de interpretación en el que el texto es un recurso. Mercer (1995) lo definió como el lenguaje en su contexto social, utilizado para llevar a cabo la vida social e intelectual de una comunidad. Si amoldamos estas definiciones al discurso político, se puede plantear que es el proceso de construcción de un mensaje que evocará un argumento conforme a la ideología del orador, ya que según Van Dijk (1980), uno de los aspectos obvios del discurso es que una ideología puede manifestarse a sí misma con el fin de buscar la aprobación del público que, dependiendo de las circunstancias políticas, sociales y económicas y su dinámica en una sociedad determinada, generan un patrón de comportamiento comunicacional basado en los temas de interés relevantes para la sociedad o ciertos grupos sociales, cuya efectividad residirá en la aceptación y podrá ser replanteado si no presenta el impacto deseado.

Lo normal en el discurso político es deteriorar la imagen del otro, por lo que la descortesía es la estrategia predominante (Muñoz, 2014). Esto es determinado por el lenguaje utilizado, aquel que brinda la oportunidad al emisor o receptor de otorgar los significados de un texto de acuerdo a sus propias experiencias y características lingüísticas (Ríos, 2015), en este tipo de lenguaje se busca legitimar al «Yo» en sus acciones y actuaciones políticas, mientras se deslegitima al «Otro» desde sus actuaciones y acciones y, a la vez, propiciar la polarización entre los grupos que apoyan o adversan al líder (León, Molero y Chirinos, 2011). Esta polarización se debe principalmente:

[...] a la preferencia del ingroup y el rechazo del outgroup, la autopresentación positiva y la asociación de "nuestro grupo" con todas las buenas cosas y "su grupo" (de ellos) con todas las malas cosas, vista a través de una definición de las ideologías como un esquema básico sujeto-grupo de cognición social, es la característica central de toda ideología que se encuentra marcada en la estructura del discurso, antes que otra cosa por los pronombres personales y posesivos (nosotros, ellos, de nosotros, de ellos, nuestro, suyo, etc.), pero también por deícticos tales como aquí y allá (Van Dijk, 1996, p.33). 
Igualmente, el discurso político pretende persuadir, por tanto, la mayoría de los mensajes están orientados en mayor medida hacia los indecisos (Dorna, 1993). En este sentido, se pueden presentar fenómenos con respecto a la predisposición que tenga el público al que va dirigido el mensaje, tales como: i) el efecto Bandwagon o de «arrastre» ${ }^{1}$,donde las personas con sus preferencias políticas e ideológicas se unen a lo que perciben como mayorías o posiciones dominantes en la sociedad y en donde los votantes se sienten atraídos por partidos o candidatos que perciben como ganadores (Schmitt-Beck, 2015), por lo que se suman a ellos durante la contienda política. ii) el efecto Underdog, este involucra el hecho de adherirse a la opción que se espera pierda, o al abstencionismo por exceso de confianza en el probable triunfo de la propia opción o por la desilusión del esperado fracaso (Crespo y Moreno, 2015).

Respecto a las redes sociales el discurso político no es homogéneo, pues al igual que en otras instancias resulta ser asimétrico con los actores que participan (Torres, 2013), puesto que no existe la limitante de uno o varios portavoces oficiales y que la rotación de la participación elimina todo escalafón interno (Sala, 2016). Sin embargo, lo mencionado no ocurre siempre ya que puede presentar, al igual que el discurso político tradicional -sobre todo en las campañas electorales-, un solo emisor responsable que tiene un rol social aceptado como líder de un partido, agrupación política, organización social o de protesta social espontánea y aquellos que cumplen el rol de seguidores o admiradores (Raiter, 2009), encargados de validar sus acciones y planteamientos.

De lo anterior se colige que el discurso político tiene como base el conductismo, ya que está "caracterizado por su concepción asociacionista; es decir, crea conocimiento al relacionar los antecedentes de una situación con sus consecuentes (estímulorespuesta)" (Leiva, 2005, p.68). En otras palabras, la forma en que se expresa un mensaje responde a una determinada cosmovisión que formará diferentes lecturas o interpretaciones (Berardi, 2003); por esta razón, los políticos buscan producir sus mensajes en torno a un proceso de identificación social, de esta manera sus contenidos contribuyen a definir antagonistas y a identificar al propio grupo de pertenencia (Cifuentes y Pino, 2018). 


\subsection{Twitter, el espacio de la comunicación política}

Debido a su naturaleza caracterizada por una relación de comunicación directa, entre pares, interactiva y en tiempo real (Moya \& Herrera, 2016), Twitter se presenta como un escenario ideal para que diferentes públicos de diversas ideologías confluyan en condiciones de igualdad (Fernández-Gómez, Hernández-Santaolalla, y Sanz-Marcos, 2018), transformándose así en la nueva «plaza pública», una moderna ágora de discusión y debate de opiniones (Cansino, Calles y Echeverría, 2016) donde se intercambian los tres discursos contradictorios de los actores que tienen legitimidad para expresarse públicamente sobre política: los políticos, los periodistas y la opinión pública (Wolton, 1998). Además, Twitter fomenta y propicia el activismo y sirve como espacio para ejercer control, supervisión y crítica de las relaciones y constantes luchas en las que intervienen las instituciones gubernamentales, los actores políticos, las fuerzas de seguridad, la prensa y la ciudadanía, generando así un proceso sinérgico-retroalimentativo que, en el caso de algunas sociedades, como la colombiana por ejemplo, rompe por lo menos, digitalmente, con el modelo centralista que presume la base de la gobernanza y el monopolio informativo imperante (Romero-Rodríguez, 2014).

No obstante, para las elecciones de la última década, el sector político ha aprovechado el potencial ofrecido por Twitter utilizándolo como un medio efectivo para el proselitismo, debido a que se ha comprobado que la interacción de los candidatos con los ciudadanos por este medio aumenta su popularidad y visibilidad, principalmente, entre sus electores prospectivos (Aharony, 2012); ya que a través de la plataforma se pide a los usuarios asistencia a los actos de campaña, su colaboración como voluntarios, realizar donaciones, que vayan a votar o que den sus opiniones (López-Meri, Marcos-García y Casero-Ripollés, 2017). En este escenario, incluso los periodistas tradicionales se entremezclan con los blogueros, con el público y los propios candidatos para ofrecer una amplia gama de comentarios electorales (Mcgregor y Mourão, 2016). 
Esto presume que Twitter puede ser usado como «instrumento de presión» por parte de políticos y generadores de opinión que buscan, mediante la exposición de contenidos, afianzar ideas en el público conforme al entorno social, en muchos casos empleando la demagogia debido a que, como sugiere Castells (2008), la información ha constituido fuentes fundamentales de poder y contrapoder, de dominación y de cambio social, en razón de que la batalla más importante que hoy se libra en la sociedad es aquella que busca adueñarse de la opinión pública, la cual varía no únicamente por los intereses del individuo, sino también según este pueda o no ser impelido a tomar partido públicamente respecto a un hecho (NoëlleNeumann, 1995).

El espacio ofrecido por Twitter fomenta un intercambio fluido de la conversación (Campos-domínguez, 2017) por lo que resulta pertinente acotar, desde una posición dicotómica, que la plataforma puede incrementar el debate político, pero también puede entorpecerlo a causa de la información fugaz y muchas veces imprecisa resultante del continuo bombardeo de contenido al que se expone el usuario; donde juega un papel concluyente la percepción, encargada de determinar la entrada de los mensajes y garantizar que la información retomada del ambiente permita la formación de abstracciones (juicios, categorías, conceptos, etc.) (Oviedo, 2004). Esta situación acarrea muchas veces que se asuma lo expuesto como verídico sin constatarlo, construyendo una concepción a priori y creando juicios de valor dentro una realidad aparente, juicios que son erróneos en la mayoría de los casos y que conllevan a acciones que repercuten en lo tangible ya que los mensajes expuestos en Twitter tienen como función captar la atención de los usuarios y movilizar sus sentimientos políticos; todo lo anterior sucede gracias a que la plataforma posee la función tecnológica necesaria para efectuar el lema de 'sigue tus intereses' de manera satisfactoria (Sala, 2016) aun cuando no se tenga claro cuáles son.

Incluso las redes sociales - como Twitter- se suman a la prensa, la radio y la televisión como otra fuente que alimenta la conversación (Moya y Herrera, 2015), de esta manera, los usuarios interactúan con otros en torno a la información emitida, convirtiendo cierto tipo de contenido en Trending Topic, especialmente, aquél que expone las preocupaciones ciudadanas, su visión de la realidad con respecto al contexto o aquel que expresa una idea complementaria o de contraste a la información expuesta. 
Por ello, una de las herramientas utilizadas en Twitter para este fin es el hashtag, el cual permite "llamar la atención de la ciudadanía sobre las tendencias más socorridas, las causas sociales en lucha y para suplir la escasez de participación política" (Sala, 2016, p.29). Con la opción del hashtag se logra hacer inferencia sobre los temas que preocupan a los ciudadanos y a los medios, haciendo de "Twitter una herramienta esencial para entender la dinámica de la agenda pública y prever los temas de mayor impacto en la agenda mediática” (Fernández, 2012, p.13).

\section{Materiales y método}

La presente investigación es de alcance exploratorio y correlacional por lo que servirá para sentar un precedente y anteceder a otras investigaciones con alcances descriptivos, correlacionales o explicativos (Hernández, Fernández y Baptista, 2014), ya que este tipo de investigaciones examinan un tema poco estudiado del cual se tiene muchas dudas o que no se ha abordado antes. Este estudio presenta un diseño mixto (cuali-cuantitativo) que permitió, mediante lo cuantitativo, la medición numérica de los datos cuantificables y su posterior análisis estadístico para establecer patrones de comportamiento y seguidamente buscar regularidades y relaciones causales entre elementos; mientras que por medio de lo cualitativo se establecieron percepciones, creencias y conceptos manifestados en el lenguaje de los participantes (Hernández et al., 2014).

Considerando que los términos «primera vuelta» $\mathrm{y}$ «segunda vuelta» corresponden, en sentido estricto, a los días en que se efectuaron los comicios de 2018, el 27 de mayo y el 17 de junio respectivamente, para efectos de síntesis se consideró apropiado que los períodos analizados en este artículo adquirieran las siguientes denominaciones:

- Primera vuelta: haciendo referencia al mes previo a los primeros comicios; del 17 de abril hasta el 27 de mayo.

- Segunda vuelta: haciendo referencia del 28 de mayo hasta el 17 de junio, día de los comicios finales. 
De este modo se tuvieron en cuenta 3858 tweets de las cuentas de los candidatos a la presidencia colombiana en el año 2018, Iván Duque (@IvanDuque) y Gustavo (@petrogustavo),durante el mes previo a la primera vuelta y la segunda vuelta electoral. Estos tweets se obtuvieron a través de las plataformas Twitonomy ${ }^{\circledR}$ y Twitter Counter ${ }^{\circledR}$ y se separaron los tweets propios de los candidatos (1890) de los retweets que realizaron los usuarios (1968), los cuales fueron excluidos de la muestra.

Asimismo, para el análisis cualitativo, se realizó una grilla de análisis de contenido a partir de los propios lexicones emergentes de una prueba piloto. Esta ficha fue puesta en análisis por un panel de expertos, resultando validada con un coeficiente de kappa de Cohen mayor a 0.70 .

\section{Resultados y comparación}

\subsection{Comportamiento cuantitativo de las cuentas y seguidores}

Del 27 de abril al 27 de mayo, Iván Duque realizó 619 tweets frente a Gustavo Petro que publicó 486, siendo la diferencia entre ambos de 133 (27,3\%). Durante el 28 de mayo al 17 de junio, Duque realizó 407 tweets mientras Petro hizo 378, una diferencia de 29 (7,67\%) entre los candidatos.

En el mes previo a la primera vuelta la cuenta @IvanDuque pasó de tener 305.696 seguidores a 348.995 un 12,4\% más (43.299 nuevos). El 28 de mayo, el número de usuarios incrementó a 363.513 seguidores (14.518 nuevos); y desde esa fecha hasta el 17 de junio la cifra cerró con 413.057 seguidores, lo que significa que hubo un incremento del 11,99\% (49544 nuevos seguidores). Durante ambos períodos Duque contó con la adición de 107.361 usuarios. Por su parte, la cuenta @petrogustavo pasó de tener 3.095.773 seguidores el 27 de abril a 3.154 .535 el 27 de mayo, lo que supone un aumento del 1,8\% que se traduce a 58.762 nuevos usuarios; mientras que el 28 de mayo, el numero de usuarios aumentó a 3.169.371 (14,836 nuevos). Desde esa fecha hasta el 17 de junio, la cifra finiquitó en 3.226.629 seguidores, representando un aumento del 1,77\% (57.258 nuevos). El total de adheridos en ambos periodos fue de 130.856 . 
Respecto al número total de tweets compartidos y el número de «me gusta» en la cuenta de @petrogustavo, se observó que la interacción de los usuarios fue mayor en la segunda vuelta. Del 27 de abril al 27 de mayo, el total de veces en lo tweets compartidos fue de 595.605 mientras que del 28 de mayo al 17 de junio fue de 844.195. Esto significa que la interacción en este último período incrementó un $29,45 \%$. En el caso de los «me gusta», la relación fue de 1.619 .570 y 2.313.718, un 30\% más en el segundo período. En la cuenta @IvanDuque hubo una disminución en el número de tweets compartidos y un aumento en los «me gusta». En el primer período, el total de veces en los tweets compartidos fue de 271.636 y para el segundo fue de 267.091, lo que supuso un decrecimiento del-1,77\%; en el número de «me gusta» la relación fue de 515.315 y 589.336, lo que refleja un aumento significativo del $12,66 \%$. Se debe considerar que el período de segunda vuelta tuvo una semana menos.

El número de personas a las que seguía Gustavo Petro tuvo un aumento en ambos períodos, pasando de tener 2.050 el 27 de abril a 2.059 el 27 de mayo, y 2.057 el 28 de mayo, donde decreció -2, a 2.132 el 17 de junio. Es posible que este incremento significativo durante la segunda vuelta se gestara mediante el «me sigues, te sigo» y también dado a que a la campaña de «Colombia Humana» se sumaron varios actores y artistas colombianos, como también personalidades académicas de trascendencia internacional como Noam Chomsky, Thomas Piketty y John Maxwell Coetzee. Mientras que en la cuenta de Duque el número de usuarios a los que seguía disminuyó levemente, pasando de tener 2.483 del 27 de abril a 2.482 el 27 de mayo, y 2.482 el 28 de mayo a 2.479 el 17 de junio; esta pequeña disminución pudo suceder por alguna aseveración expresada en alguna de las cuentas que seguía que pusiera en juicio su imagen. 


\subsection{Temáticas dialógicas imperantes en las relaciones}

En las propuestas de gobierno el candidato Ivan Duque se enfocaba en temas como economía y desarrollo, justicia y equidad (ver tablas 1 y 2 ), por ello, durante ambos períodos estos fueron los temas principales de sus contenidos en Twitter.

\begin{tabular}{lc}
\multicolumn{1}{c}{ Temas } & Porcentaje \\
\hline Economía y Desarrollo & $\mathbf{2 1 , 9 7 \%}$ \\
\hline otros & $\mathbf{1 4 , 8 6 \%}$ \\
\hline Seguridad & $\mathbf{1 1 , 9 5 \%}$ \\
\hline Justicia & $\mathbf{1 0 , 3 4} \%$ \\
\hline Equidad & $\mathbf{9 , 0 5} \%$ \\
\hline Salud y pensión & $\mathbf{8 , 2 4} \%$ \\
\hline Cornupción & $\mathbf{5 , 9 8} \%$ \\
\hline Educación & $\mathbf{5 , 8 2} \%$ \\
\hline Empleo & $\mathbf{5 , 3 3} \%$ \\
\hline Paz & $\mathbf{2 , 4 2} \%$ \\
\hline Transporte y movilidad & $\mathbf{2 , 4 2} \%$ \\
\hline Medio ambiente y cambio & $\mathbf{1 , 6 2} \%$ \\
climático &
\end{tabular}

\begin{tabular}{lc}
\multicolumn{1}{c}{ Temas } & Porcentajes \\
\hline Economia y Desarrollo & $\mathbf{2 6 , 5 4 \%}$ \\
\hline Otros & $\mathbf{1 5 , 9 7 \%}$ \\
\hline Justicia & $\mathbf{1 0 , 0 7 \%}$ \\
\hline Equidad & $\mathbf{8 , 1 1 \%}$ \\
\hline Seguridad & $\mathbf{7 , 6 2 \%}$ \\
\hline Cornupción & $\mathbf{7 , 1 3 \%}$ \\
\hline Empleo & $\mathbf{6 , 8 8 \%}$ \\
\hline Salud y pensión & $\mathbf{5 , 6 5 \%}$ \\
\hline Paz & $\mathbf{3 , 4 4 \%}$ \\
\hline Medio ambiente y cambio & $\mathbf{3 , 4 4 \%}$ \\
climático & \\
\hline Educación & $\mathbf{3 , 4 4 \%}$ \\
\hline Transporte y movilidad & $\mathbf{1 , 4 7 \%}$
\end{tabular}

Tabla 1. Porcentaje de temas a los que hizo alusión Iván

Tabla 2. Porcentaje de temas a los que hizo alusión Iván Duque en su cuenta durante el 27 de abril al 27 mayo.

en su cuenta Duque durante el 28 de mayo al 17 junio

Nota: "Otros" corresponde al posicionamiento de su imagen: saludos, agradecimientos, convocatorias, invitaciones, aclaraciones y réplicas.

Por su parte, los temas que encabezaron la campaña en Twitter de Gustavo Petro durante el 27 de abril al 27 de mayo fueron economía y desarrollo, corrupción y recursos naturales; mientras que durante la segunda vuelta fueron paz, corrupción y equidad. Se infiere que hubo un cambio en ciertos contenidos para lograr una mayor aceptación, aun cuando mantuvo el tema de la corrupción como bandera de su campaña (ver tablas 3 y 4). 


\begin{tabular}{lc}
\hline \multicolumn{1}{c}{ Temas } & Porcentaje \\
\hline Economía y Desarrollo & $\mathbf{1 5 , 2 3 \%}$ \\
\hline Corrupción & $\mathbf{1 2 , 7 6 \%}$ \\
\hline Otros & $\mathbf{1 1 , 5 2 \%}$ \\
\hline $\begin{array}{l}\text { Medio ambiente y cambio } \\
\text { climático }\end{array}$ & $\mathbf{1 0 , 0 8 \%}$ \\
\hline Seguridad & $\mathbf{7 , 6 1 \%}$ \\
\hline Justicia & $\mathbf{7 , 6 1 \%}$ \\
\hline Equidad & $\mathbf{7 , 4 1 \%}$ \\
\hline Salud y pensión & $\mathbf{7 , 0 0 \%}$ \\
\hline Educación & $\mathbf{6 , 5 8 \%}$ \\
\hline Empleo & $\mathbf{6 , 3 8 \%}$ \\
\hline Paz & $\mathbf{4 , 7 3 \%}$ \\
\hline Transporte y movilidad & $\mathbf{3 , 0 9 \%}$ \\
\hline
\end{tabular}

Tabla 3. Porcentaje de temas a los que hizo alusión Gustavo

Petro en su cuenta durante el 27 de abril al 27 mayo.

\begin{tabular}{lc}
\hline \multicolumn{1}{c}{ Temas } & Porcentaje \\
\hline Paz & $\mathbf{1 3 , 7 4 \%}$ \\
\hline Corrupción & $\mathbf{1 3 , 0 3} \%$ \\
\hline Otros & $\mathbf{1 1 , 8 5} \%$ \\
\hline Equidad & $\mathbf{1 0 , 9 0 \%}$ \\
\hline Economia y Desarrollo & $\mathbf{1 0 , 4 3} \%$ \\
\hline Seguridad & $\mathbf{8 , 5 3} \%$ \\
\hline Justicia & $\mathbf{8 , 5 3} \%$ \\
\hline Medio ambiente & $\mathbf{6 , 1 6} \%$ \\
cambio climático & \\
\hline Educación & $\mathbf{5 , 6 9 \%}$ \\
\hline Salud y pensión & $\mathbf{5 , 2 1} \%$ \\
\hline Empleo & $\mathbf{4 , 7 4} \%$ \\
\hline Transporte y movilidad & $\mathbf{1 , 1 8} \%$ \\
\hline Tabla 4. Por & \\
\hline
\end{tabular}

Tabla 4. Porcentaje de temas a los que hizo alusión Gustavo Petro en su cuenta durante el 28 de abril al 17 junio.

Nota: "Otros" corresponde al posicionamiento de su imagen: saludos, agradecimientos, convocatorias, invitaciones, aclaraciones y réplicas.

\subsection{Demonización de la otredad}

En Colombia, la radicalización de los comicios de 2018 encontró su sustento en el uribismo y el antiuribismo que, a su vez, adquirió en los seguidores de Gustavo Petro, el nombre de petrismo. El uribismo representado por Iván Duque simboliza, grosso modo, una doctrina populista resultado de un proceso articulatorio de diversos grupos y exigencias sociales concertadas en torno de una demanda popular, la "recuperación de la patria", hasta instaurarse como el núcleo aglutinador de dichas diferencias (López, 2016).

El uribismo maneja un discurso político sencillo, directo, corriente, popular y moralista (Fierro, 2014), en donde los buenos lo apoyan, y los malos son comunistas disfrazados que pretenden entregar la patria a las guerrillas y a la delincuencia (“Más allá del liderazgo de Uribe", 2010), asumiendo la política como una lucha moral y frontal contra un enemigo; no acepta el diálogo ni la negociación, conceptos fundamentales de la democracia liberal (Fierro, 2014). 
Se fundamenta en el neoliberalismo pretendiendo anular la intervención del estado en el mercado conllevando a su privatización y posteriormente a la explotación, transformación y distribución de los recursos por parte de empresas transnacionales. Plantea, igualmente, un modelo de salud y pensión administrado a través de contratos por entidades no reguladas y la tercerización laboral.

El petrismo, también populista y de discurso provocador, pretende implementar un modo de gobierno redistributivo que favorezca al pueblo en contra de la oligarquía y la burguesía (López, 2016), enfocado hacia una política social y proteccionista en lo que al mercado se refiere. En la confrontación de ambas ideologías, las personas que no se matricularon en alguna de estas recibieron el peyorativo de "tibios", manifestando los síntomas de la debilidad, la vacilación, la cobardía o la ecuanimidad (Maya, 2012), especialmente, después de finalizada la primera vuelta electoral y la eliminación de los otros candidatos.

Ambos aspirantes aprovecharon la polarización existente y forjaron discursos demonizantes, principalmente Petro, mediante los cuales buscaron menospreciar a su adversario y hacerlo ver ante la opinión pública como inaceptable y moralmente inferior, criminalizando sus puntos de vista, radicalizando el discurso y distrayendo a la sociedad; mientras las ideas del emisor se justificaban considerándose acertadas (Romero-Rodríguez, Aguaded y Gadea, 2015). De esta forma, el sujeto demonizado es señalado dicotómicamente como un ente objeto de desagrado o nocividad para la humanidad con el fin de obtener la favorabilidad de la opinión pública (Romero-Rodríguez y Römer, 2016).

Por ello, uno de los aspectos a resaltar es que Duque usó la crisis que sufre Venezuela como elemento referencial propagandístico al asociarla de manera indirecta el modelo de gobierno que proponía Petro. En esta línea se encontraron durante el 27 de abril al 27 de mayo 5 tweets, de 18 que contenían la palabra Venezuela, que hicieron esta referencia. Durante la segunda vuelta, los comentarios concernientes a la situación venezolana se redujeron a 5; sin embargo, no hizo alusión a su adversario sino que manifestó solidaridad con el país vecino. Cabe anotar que la cuenta de Petro fue mencionada el 28 de mayo en aras de la realización de un debate electoral. 


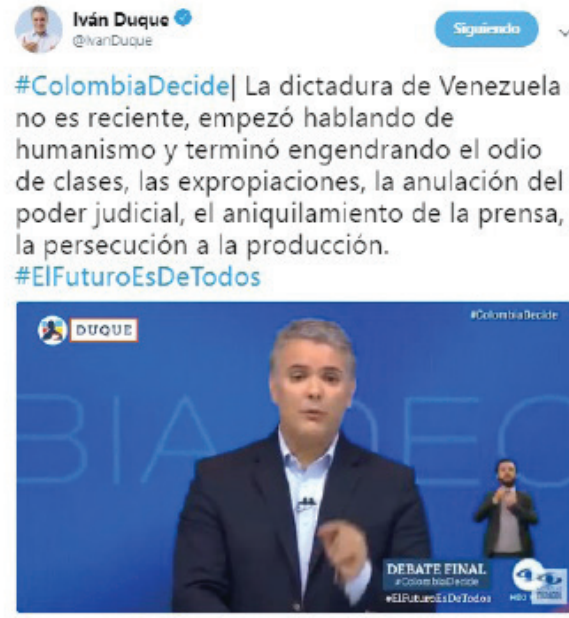

21:37- 25 mos, 2018

Iván Duque

Qhar Dugue

\#SanMateol En Colombia no podemos dejar que vengan con el discurso que arruinó a Venezuela, el discurso del odio de clases. Tenemos que ser firmes y ganar esta elección en primera vuelta. \#ElFuturoEsDeTodos

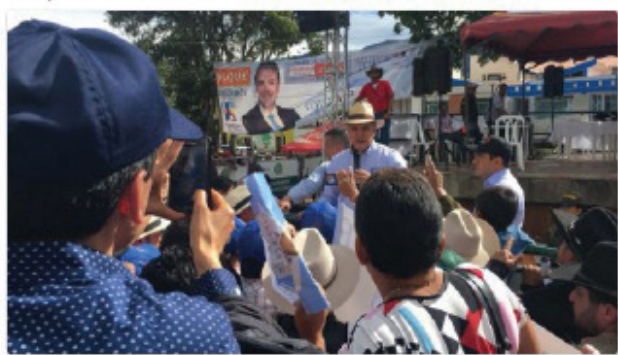

14.51 .16 mey. 2018

Gráfico 1. Ejemplos de tweets de Duque asociando indirectamente al modelo propuesto por Petro con la crisis venezolana.

Por otro lado, Petro utilizó la desacreditación directa, aseverando en varios de sus comentarios que Duque representaba la corrupción. Duque fue mencionado 68 veces en ambos períodos ( 29 y 49, respectivamente).

Gustavo Petro

@petrogustavo

Lo que se enfrenta el domingo en las urnas es la ciudadania contra la corrupción. Alguna prensa le llama a la corrupción la maquinaria para tapar su olor. A la corrupción la representa Duque y Vargas

16:06 - 24 may. 2018
Siguiendo $\vee \quad$ Gustavo Petro

@etrogustavo

El voto en blanco sólo es un voto por Uribe/Duque

Esto es entre la maquinaria corrupta que nos llevó a la violencia, a la desigualdad social, a la metástasis de la corrupción: Hidroituango y Odebrecht y las ciudadanías libres que nos pueden llevar a la Demicracia plena y la Paz 4:28 - 31 may. 2018

Gráfico 2. Ejemplos de tweets de Petro con aseveraciones desacreditantes. 


\subsection{Estrategias de viralización de los mensajes}

El uso del hashtag (\#) fue predominante en la campaña de Duque durante ambos períodos. Del 27 de abril al 27 de mayo, el 95\% de los tweets (593) iba acompañado de por lo menos un hashtag. Para la segunda vuelta, el 94.4\% de sus tweets (384) tenía al menos una etiqueta. Una de las estrategias que utilizó fue el uso de \#ElFuturoEsDeTodos, eslogan de su campaña, sin discriminar temas ni contenidos. Además utilizó etiquetas de algunas de las ciudades principales a las que se dirigió en su recorrido por el país para fomentar la participación de sus seguidores habitantes de estas zonas (ver tablas 5 y 6 ).

\begin{tabular}{ccc}
\hline No & Hashtag & No de uso \\
\hline 1 & \#ElFuturoEsDeTodos & $\mathbf{3 2 7}$ \\
\hline 2 & \#DuqueConLasRegiones & $\mathbf{5 6}$ \\
\hline 3 & \#Bogotá & $\mathbf{4 9}$ \\
\hline 4 & \#DuqueConElPacifico & $\mathbf{2 9}$ \\
\hline 5 & \#Cali & $\mathbf{2 4}$
\end{tabular}

Tabla 5. Los cinco hashtags más usados en la cuenta de Iván Duque durante el periodo 27 de abril al 27 de mayo.

\begin{tabular}{lcc}
\hline No & Hashtag & No de uso \\
\hline 1 & \#ElFuturoEsDeTodos & $\mathbf{2 3 9}$ \\
\hline 2 & \#ConElFuturoNoSeJuega & $\mathbf{7 1}$ \\
\hline 3 & \#Cali & $\mathbf{2 2}$ \\
\hline 4 & \#DuqueEnCableNoticias & $\mathbf{2 1}$ \\
\hline 5 & \#Bogotá & $\mathbf{1 6}$ \\
\hline
\end{tabular}

Tabla 6. Los cinco hashtags más usados en la cuenta de Iván Duque durante el 28 de mayo al 17 junio.

Gustavo Petro no le dio mucha importancia a los hashtags. Durante el 27 de abril al 27 de mayo solo un 11.1\% (54) de sus tweets estuvieron acompañados de una o más etiquetas. Igual ocurrió en la segunda vuelta, donde solo utilizó las etiquetas en un 5.3\% (20) de sus tweets. En ambos períodos los hashtags que más utilizó Petro estaban enfocados a generar confianza en su imagen y convocar gente a las manifestaciones y eventos de su campaña (ver tablas 7 y 8).

\begin{tabular}{ccc} 
No & Hashtag & No de uso \\
\hline 1 & \#PetroPresidente & $\mathbf{2 8}$ \\
\hline 2 & \#YoVotoPetro & $\mathbf{2 4}$ \\
\hline 3 & \#PetroEsPasión & $\mathbf{1 5}$ \\
\hline 4 & \#PetroEnPrimeraVuelta & $\mathbf{1 0}$ \\
\hline 5 & \#ColombiaHumana & $\mathbf{9}$
\end{tabular}

Tabla 7. Los cinco hashtags más usados por Gustavo Petro durante el periodo 27 de abril al 27 de mayo.

\begin{tabular}{lcc} 
No & Hashtag & No de uso \\
\hline 1 & \#LeVamosADarLavuelta & $\mathbf{5}$ \\
\hline 2 & \#ColombiaEnVivoConPetro & $\mathbf{3}$ \\
\hline 3 & \#ConPetroElSueñoSeAgranda & $\mathbf{1}$ \\
\hline 4 & \#PetroEsElCambioDeVerdad & $\mathbf{1}$ \\
\hline 5 & \#YoConfioEnPetro & $\mathbf{1}$
\end{tabular}

Tabla 8. Los cinco hashtags más usados de la cuenta de Gustavo Petro durante el 28 de abril al 17 junio. 


\section{Discusión y conclusiones}

En las elecciones de Colombia 2018, Twitter jugó un papel importante pero no determinante. Esto se deduce con base en las cifras que se obtuvieron de la elección final donde Iván Duque fue electo presidente de la República de Colombia con 10.373.080 votos; mientras que su cuenta tenía 413.057 seguidores. Por otro lado, Gustavo Petro perdió con 8.034.189; mientras su cuenta ostentaba 3.226.629 seguidores. Esto indica que, aunque Twitter cada vez tiene más fuerza en las estrategias de las campañas político-electorales de Colombia, por ser un medio de autopromoción y difusión (Enli \& Simonsen, 2017), aún queda en segundo plano frente a la metodología tradicional de proselitismo. Sin embargo, cabe destacar que es un medio efectivo para que los candidatos comprendan algunas características y preferencias de sus seguidores.

Otro aspecto que se infiere es que durante los períodos analizados ambos candidatos mantuvieron en Twitter una relación unidireccional con sus seguidores, asumiéndolos como consumidores pasivos del contenido presentado. La estrategia de no establecer una conversación con el público en Twitter se debía a que el ambiente de deliberación electoral tenía niveles de polarización muy altos, ya que nunca antes la política colombiana había sido tan hostil en el uso del discurso político (Broda, 2018), y cualquier paso en falso podía generar escándalo y deformar la imagen del candidato en la opinión de sus seguidores favoreciendo al adversario.

Los candidatos no utilizaron de primera mano memes seguramente por la noción que adquirieron de ser expresiones demasiado informales e infantilizar y ridiculizar el lenguaje y el discurso político. Tampoco se evidenció en ninguno de los candidatos la realización de directos a través de Twitter, pero sí utilizaron la plataforma para anunciarlos en otras redes sociales, predominantemente Facebook, posiblemente por desconocimiento de esta función en Twitter o, simplemente, porque no la consideraron necesaria o estratégica. Sin embargo, sí se verifica que utilizaron elementos audiovisuales en la mayoría de sus publicaciones para acompañar el contenido textual. 
Gustavo Petro enfocó parte de su campaña para afirmarse y desacreditar (Berger y Luckmann, 1968) a Duque y a varios miembros de su partido, además de aplicar, en mayor medida lo planteado por Van Dijk (1996) sobre la asociación de cualquier acto positivo con su grupo y lo negativo con los que no pertenezcan a él, legitimando de esta manera el «yo» (León et al. 2011) y consiguiendo con esto una mayor radicalización en la posición, tanto de sus partidarios como en los de Duque.

Por su parte y como se mencionó antes, Iván Duque asoció indirectamente el modelo de gobierno venezolano con varias propuestas de Petro, quien, según él y su bancada, instauraría modos productivos que llevaron a la crisis a países que en algún momento se consideraron socialistas o alguna de sus vertientes e implantaría un régimen político-social antidemocrático y totalitarista. Se deduce entonces que utilizó tres de las 10 estrategias de manipulación mediática propuestas por ): i) La distracción, al referirse al modelo de gobierno venezolano y compararlo con lo planteado por Petro; ii) la gradualidad, aprovechándose de la construcción preconcebida de un enemigo externo transformado en una ideología "nefasta" que asechaba a Colombia y iii) el uso del aspecto emocional frente a la reflexión, cuando aseveraba que Colombia terminaría con una crisis igual a la de Venezuela.

Considerando las posiciones ideológicas de Gustavo Petro (Centro-izquierda progresista) e Iván Duque (Centro-derecha) y definiendo ideología con base en Van Dijk (1980) como un sistema cognitivo relacionado con cuestiones socialmente relevantes en la vida del individuo que no residen únicamente en conocimientos y creencias, sino también en opiniones y actitudes, muchas veces compartidas por grupos, utilizadas para la interpretación de acontecimientos, acciones o para comprensión de un determinado discurso, se considera posible, a través Twitter, ubicar en qué espectro político se encuentra un candidato mediante la agrupación de sus publicaciones, si este hace énfasis a aspectos del contenido vinculantes a alguna posición, por ejemplo: 
Duque se refirió en mayor media a la economía y el desarrollo, proponiendo, grosso modo, mayores beneficios económicos a las empresas para fomentar la inversión extranjera, reducción del gasto público y apertura económica.

Petro se comprometía a desarticular la corrupción en Colombia para generar una política de bienestar social en el que varios servicios, como la educación y la salud, fueran subsidiados por el Estado, también se comprometía a eliminar la tercerización laboral mejorando los derechos y la calidad de vida de los trabajadores.

A raíz de ello se encontró un aspecto interesante, la estratificación socioeconómica en el imaginario colectivo de algunos sectores sociales atribuida a los candidatos; Duque era asociado con empresarios que ostentaban riqueza y poder; y a Petro lo relacionaban con personas de bajos recursos, campesinos y estudiantes. Asimismo, se coligaron con actores ilegales del conflicto armado colombiano; Duque con el paramilitarismo debido a las investigaciones que adelanta la justicia colombiana contra el jefe de su partido político, Álvaro Uribe Vélez, y varios miembros de esta tolda por nexos con grupos paramilitares; mientras que Petro, por su pasado y su posición ideológica, con las guerrillas, principalmente con las disidencias de las FARC.

Al revisar varios comentarios de usuarios en los tweets de Duque, se encontró como resultado emergente una fuerte oposición al condicionamiento que tiene la democracia en Colombia, caracterizada como defectuosa (Velásquez \& Rojas, 2017), con el modelo neoliberal establecido por un proceso posdemocrático; esto significa que Duque encarnaba para sus opositores, la entrega de la gestión colectiva a los «expertos», la externalización de decisiones, la burocratización de los partidos, el gobierno de las multinacionales y el despotismo de la economía financiera sobre la realidad social (Monedero, 2012). 


\section{Notas}

${ }^{1}$ Este efecto está muy vinculado a la «Espiral del silencio» de la opinión pública, explicado por Elisabeth Nöelle-Neumann como un fenómeno social en el que las personas temen quedar aisladas de la noción de grupos por tener opiniones contrarias a la mayoría social.

\section{Referencias}

Aharony, N. (2012). Twitter use by three political leaders: An exploratory analysis. Online Information Review, 36(4), 587-603. Recuperado de https://doi.org/10.1108/14684521211254086

Allcott, H., \& Gentzkow, M. (2017). Social Media and Fake News in the 2016 Election. Journal of Economic Perspectives, 31(2), 211-236. Recuperado de https://doi.org/10.1257/jep.31.2.211

Barreto, I., López, W., \& Sánchez, L. (2013). Cognitive framing of the political culture. An analysis from the social networks in the Internet ( Twitter ). Revista Interamericana de Psicología/Interamerican Journal of Psychology, 47(3), 383-394.

Binder, A. (1992). La sociedad fragmentada. Nueva Sociedad, (111), 100-108.

Bauman, Z. (2002). Modernidad líquida (1st ed.). México: FCE. https://doi.org/ EBACBAUM

Berardi, L. (2003). El Análisis Crítico del Discurso: Perspectivas

Latinoamericanas. (Frasis, Ed.) (1st ed.). Santiago: Frasis. 
Berger, P. L., \& Luckmann, T. (1968). La sociedad como realidad objetiva. In La Construcción Social De La Realidad (1st ed., pp. 66-163). Buenos Aires: Amorrortu.

Borda, S. (2018). Presidenciales en Colombia: ¿polarización o deterioro de la conversación política? Recuperado de https://goo.gl/a6vmNg

Cansino, C., Calles, J., \& Echeverría, M. (2016). Del Homo Videns al Homo Twitter: democracia y redes sociales. (C. Cansino, J. Calles, \& M. Echeverría, Eds.) (1st ed.). Puebla: Benemérita Universidad Autónoma de Puebla.

Castells, M. (2008). Comunicación, poder y contrapoder en la sociedad red. Los medios y la política. Telos: Cuadernos de Comunicación e Innovación, (74), 13-24.

Cifuentes, C. F., \& Pino, J. F. (2018). Conmigo o contra mí: análisis de la concordancia y estrategias temáticas del Centro Democrático en Twitter. Palabra Clave - Revista de Comunicación, 21(3), 885-916. https:// doi.org/10.5294/pacla.2018.21.3.10

Constitución política de Colombia. (1991). Artículo 190, Del Presidente de la República. 40a Ed. Legis.

Crespo, I., \& Moreno, C. (2015). Los efectos de la comunicación política en el comportamiento electoral. Revista de Derecho Electoral, (20), 76-110. Recuperado de https://goo.gl/jxNU2g

Dahl, R. (1992). La democracia y sus críticos. Barcelona: Paidós.

Dorna, A. (1993). Estudios Sobre El Discurso Político. Psicología Política, (6), 117-128. Recuperado de https://goo.gl/3gRGhD 
Enli, G., \& Simonsen, C. (2017). "Social Media Logic” Meets Professional Norms: Twitter Hashtag Usage by Journalists and Politicians. Information, Communication \& Society., 21(8), 1081-1096. https://doi.or g/10.1080/1369118x.2017.1301515

Fairclough, N. (1989). Language and Power (1st ed.). London: Logan.

Fernández-Gómez, J., Hernández-Santaolalla, V., \& Sanz-Marcos, P. (2018). Influencers, marca personal e ideología política en Twitter. Cuadernos. Info, 0(42), 19-37. https://doi.org/10.7764/CDI.42.1348

Fernández, C. B. (2012). Twitter y la ciberpolítica. Disertaciones: Anuario Electrónico de Estudios En Comunicación Social, 5(1), 9-24.

Fierro, M. (2014). Álvaro Uribe Vélez, populismo y neopopulismo. Analisis Político, 27(81), 127-147. Recuperado de https:/goo.gl/YX69Zk

Galleguillo, R. E. (2015). Comunicación interactiva y poder ciudadano en la ciber-democracia. Universidad Complutense de Madrid.

García-Dussán, E. (2011). El delirio mockusiano : tecnologías de la comunicación y acción. Revista Tendencias y Retos, (16), 291-305. Recuperado de https://goo.gl/FuT91B

Hernández, R., Fernández, C., \& Baptista, M. (2014). Metodología de la investigación (6th ed.). México D.F.: McGraw-Hill. https://doi.org/ISBN978-92-75-32913-9

Leiva, C. (2005). Conductismo, cognitivismo y aprendizaje. Tecnología En Marcha, 18(1), 66-74.

León, F., Molero, L., \& Chirinos, A. (2011). El discurso político en Latinoamérica. Análisis semántico-pragmático. QUÓRUM ACADÉMICO, $8(15)$. 
Levy, P. (2004). Inteligencia Colectiva. Por una antropología del ciberespacio.

Whashinton D.C.: Unidad de Promoción y Desarrollo de la Investigación de la Organización Panamericana de la Salud. https://doi.org/10.1017/ CBO9781107415324.004

López, Á. (2016). El Uribismo y su carácter Populista. Una reconstrucción de sus Condiciones de Posibilidad. Virajes, 18(1), 87-107. https://doi. org/10.17151/rasv.2016.18.1.5

López-Meri, A., Marcos-García, S., \& Casero-Ripollés, A. (2017). What do politicians do on Twitter? Functions and communication strategies in the Spanish electoral campaign of 2016. El Profesional de La Información, 26(5), 795. https://doi.org/10.3145/epi.2017.sep.02

Más allá del liderazgo de Uribe. (2010). Recuperado de https://goo.gl/Bs2Y5D

Mcgregor, S. C., \& Mourão, R. R. (2016). Talking Politics on Twitter :

Gender, Elections , and Social Networks. Social and Media, 2(3). https:// doi.org/10.1177/2056305116664218

Mercer, N. (1995). The Guided Construction of Knowledge: Talk Amongst Teachers and Learners (1st ed.). Clevedon: Multilingual Matters.

Monedero, J. C. (2012). Posdemocracia? Frente al pesimismo de la nostalgia, el optimismo de la desobediencia. Nueva Sociedad, (240), 68-86. Recuperado de http://nuso.org/media/articles/downloads/3881_1.pdf

Moya, M., \& Herrera, S. (2015). Cómo puede contribuir Twitter a una comunicación política más avanzada. Arbor, 191(774). https://doi.org/ http://dx.doi.org/10.3989/arbor.2015.774n4012 
Muñoz, M. M. (2017). Privacidad y procesado automático de datos personales mediante aplicaciones y bots.

Noëlle-Neumann, E. (1995). La espiral del silencio. (M. Cubis, Ed.) (Paidos). Barcelona: Paidos.

Oviedo, G. L. (2004). La definición del concepto de percepción en psicología con base en la teoría gestalt. Estudios Sociales, 18(18), 89-96. https:// doi.org/10.7440/res47.2013.09 RESUMEN

Raiter, A. (2009). Hablo y entiendan: creencias, presuposición e interdiscurso en los actos de Cristina Fernández de Kirchner. Oralia, (12), 73-96.

Ríos, I. (2015). El lenguaje: Herramienta de reconstrucción del pensamiento. Razón y Palabra, (72), 1-25. Recuperado de https://goo.gl/P8Vp2b

Romero-Rodríguez, L.M. (2014). Pragmática de la desinformación: Estratage mas e incidencia de la calidad informativa de los medios. Huelva: Universidad de Huelva.

Romero-Rodríguez, L. M., Aguaded, I., \& Gadea, W. (2015). De la demonización a la polarización: un análisis desde el discurso digital del gobierno y la oposición venezolana. Argos, 32(62), 97-117.

Romero-Rodríguez, L. M., \& Römer, M. (2016). Proceso de demonización de la oposición política en los hitos discursivos de Hugo Chávez según la prensa digital. Temas de Comunicación, (32), 95-124.

Sala, J. (2016). Transformando La Nación Por Twitter En El Modelo De La Democracia Líquida, (4), 13-38.

Schmitt-Beck, R. (2015). Bandwagon Effect. In The International Encyclopedia of Political Communication (1st ed., pp. 57-60). Wiley Blackwell. 
Timsit, S. (2002). Estrategias de manipulación. Retrieved from https://goo.gl/ $\mathrm{A} 8 \mathrm{hCn} 7$

Torres, L. (2013). Los mecanismos de lo político en las redes sociales de internet. Aposta, 58(58), 1-27. Retrieved from https://goo.gl/erSJis

Van Dijk, T. a. (1996). Análisis del discurso ideológico. Versión 6, 10, 15-43.

Van Dijk, T. a. (1980). Algunas notas sobre la ideología y la teoría del discurso. Semiosis, 5, 37-53. Retrieved from https://goo.gl/24g7sX

Velásquez, A., \& Rojas, H. (2017). Political Expression on Social Media: The Role of Communication Competence and Expected Outcomes. Social and Media, 3(1). https://doi.org/10.1177/2056305117696521

Wolton, D. (1998). La comunicación política: construcción de un modelo. In J. M. Ferry (Ed.), El nuevo espacio público (1st ed.). Barcelona: Gedisa. 